\title{
TRÉMATOdES DES GOÉlANDS DE L'ILE DE RIOU
}

\section{Par Jean TIMON-DAVID}

L'île de Riou constitue un petit territoire extrêmement accidenté, long de $2.500 \mathrm{~m}$. et large de $600 \mathrm{~m}$., qui émerge à $3.500 \mathrm{~m}$. de la côte, au sud de Marseille. Les falaises très escarpées, les aiguilles et les corniches de la face sud sont fréquentées en permanence par une colonie de Goélands à pieds jaunes (Larus argentatus michaellis Naumann), qui trouve sur ces rochers un biotope très favorable. Ces Oiseaux nidifient régulièrement sur l'ìle; ils se procurent une alimentation abondante aux dépens de la riche faune littorale du voisinage et des apports de l'égout qui débouche à proximité, dans la calanque de Cortiou.

Depuis plusieurs années, je poursuis des recherches sur les parasites de cette colonie et j'ai pu me procurer un matériel d'une certaine importance. Grâce à la proximité de Marseille, les Oiseaux ont pu être disséqués au laboratoire quelques heures seulement après leur mort, dans de bonnes conditions de technique et de fraîcheur : on sait, en effet, combien est rapide la décomposition des viscères chez les Oiseaux de mer. Il m'est particulièrement agréable de remercier ici M. l'Administrateur-Général Le Cerf, Directeur de l'Inscription maritime à Marseille, pour les autorisations qu'il a bien voulu m'accorder à l'occasion de mes chasses à Riou.

J'ai recueilli chez les Goélands de Riou un grand nombre d'Helminthes. Les statistiques que j'ai dressées permettent de se faire une idée de l'épidémiologie locale et des variations qui s'observent suivant l'âge de l'hôte. J'apporte ici les résultats concernant les Trématodes seulement : sur 13 espèces obtenues, 3 sont nouvelles et plusieurs sont signalées pour la première fois en France. 58 Goélands ont été examinés (41 adultes des deux sexes et 17 jeunes).

AnN. de Parasitologie, $T$. XXX, $x^{\circ} 5-6 .-1955$. 


\section{Famille ECHINOSTOMATIDAE Looss 1902}

APORCHIS MASSILIENSIS nov. sp.

Description. - Corps extraordinairement allongé (fig. 1), cylindrique, finement strié transversalement et recouvert d'écailles serrées. L'extrémité postérieure est toujours plus ou moins incurvée en crosse; l'allure générale rappelle celle d'un petit Cestode. La longueur totale varie de 14 à $41 \mathrm{~mm}$. La plus grande largeur $(1,05$ à $1,5 \mathrm{~mm}$.) se situe vers le tiers postérieur.

Ventouse orale très petite $(90$ à $150 \mu)$. L'acétabulum, très rapproché de l'extrémité antérieure, est logé dans une dépression délimitée par un repli des téguments ; son diamètre est compris entre 350 et $360 \mu$. Le plateau céphalique, long de $500 \mu$, est profondément échancré ventralement. La garniture de spicules, si rarement intacte chez les espèces de ce genre, était parfaitement conservée chez certains sujets qui ont pu être examinés encore vivants. J'ai dénombré 43 spicules, disposés sur deux rangs, le long des bords de l'échancrure, puis sur un seul rang avec interruption sur les côtés (fig. 2). Les spicules de l'échancrure sont beaucoup plus gros que les autres, atteignant $32 \times 15 \mu$; ceux qui suivent, sur les côtés, sont plus petits $(15 \times 7 \mu)$; ils diminuent progressivement en s'éloignant de l'échancrure et finissent par disparaître. Le bord terminal du plateau est armé d'une rangée de très petits spicules $(7 \times 3 \mu)$, au nombre de 12 , régulièrement alignés.

Les écailles cuticulaires, qui recouvrent entièrement le corps, sont disposées suivant des lignes transversales qui simulent une métamérisation. La cuticule est d'une épaisseur assez uniforme : $8 \mu$ en moyenne. Intérieurement, elle est tapissée par une étroite tunique de fibres musculaires concentriques $(3 \mu)$. Contrairement à ce qu'a observé Fuhrmann (1915) chez A. segmentatus, cette couche conserve partout la même épaisseur, sans se renforcer dans la région testiculaire. En dedans se trouve une seconde tunique musculaire plus robuste, formée de faisceaux longitudinaux séparés les uns des autres et qui dessinent sur les coupes transversales des ébauches de cloisons radiaires. J'ai noté une asymétrie très accusée dans le développement de ces faisceaux longitudinaux : ceux qui occupent la région dorsale et latérale droite sont beaucoup plus robustes que les autres (fig. 3). Cette disposition est évidemment responsable de l'aspect incurvé que présente régulièrement l'extrémité postérieure du ver. 


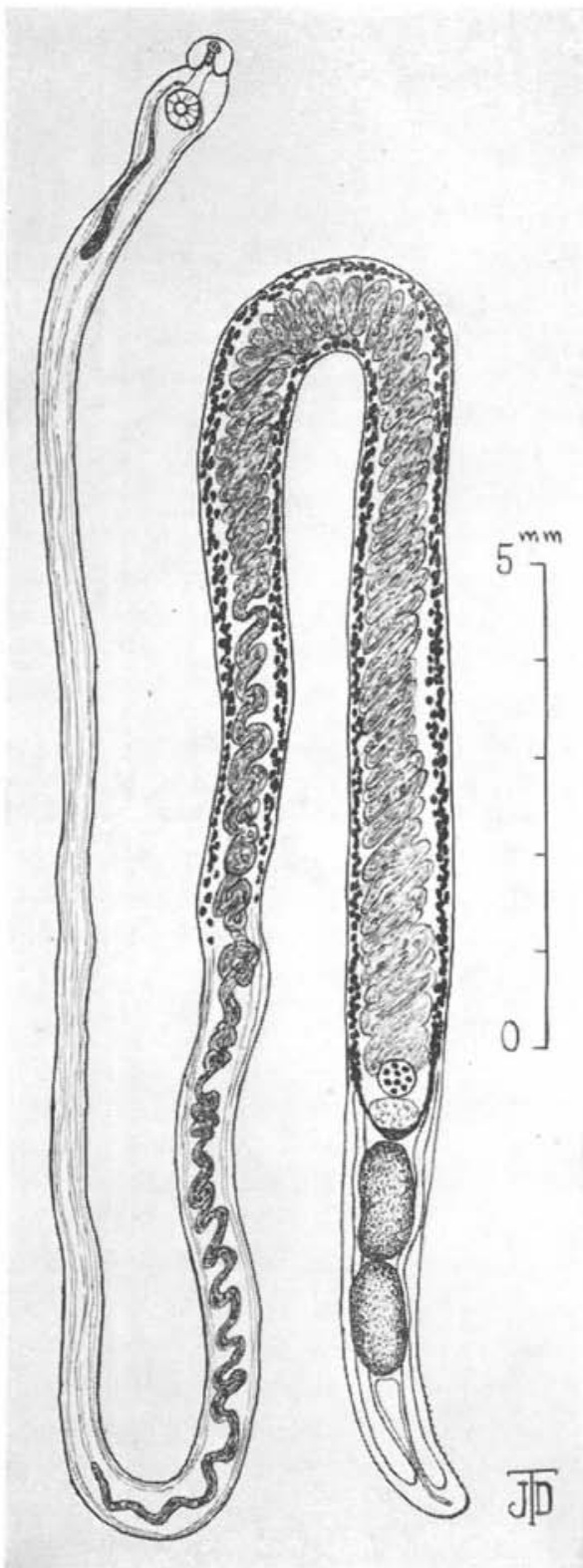

Fin. 1. - Aporchis massiliensis nov. sp.
Le parenchyme constitue un réseau à mailles polygonales assez régulières, surtout dans la portion qui entoure les branches intestinales ; les noyaux sont situés aux points de jonction. Ce parenchyme est parcouru par un troisième système de fibres musculaires qui se présentent sur les coupes tranversales sous l'aspect d'une sorte de couronne passant en dehors du tube digestif. Comme ceux qui forment la couche longitudinale sous-cuticulaire, ces faisceaux accusent une asymétrie très nette: ils sont beaucoup plus épais et plus robustes dans la région dorso-latérale droite.

Tube digestif: Le pharynx, accolé à la ventouse orale, mesure $165 \times 110 \mu$ chez un sujet de $24 \mathrm{~mm}$. L'œsophage atteint $275 \mu$ chez le même individu; le point de bifurcation se situe au niveau du bord antérieur de lacétabulum. Chaque cæcum descend en passant en dedans des vitellogènes, contourne extérieurement l'ovaire et les deux testicules, puis la vessie et se termine non loin de l'extrémité postérieure.

Organes génitaux: Les testicules sont situés l'un au-devant de l'autre dans la partie postérieure du corps 
$\left(1 / 6^{\circ}\right.$ à $\left.1 / 10^{\circ}\right)$; leur grand axe est toujours longitudinal ; ils peuvent se toucher ou être séparés par un court intervalle (100 à $300 \mu)$; leurs dimensions sont les suivantes : antérieur : longueur, 1,10 à

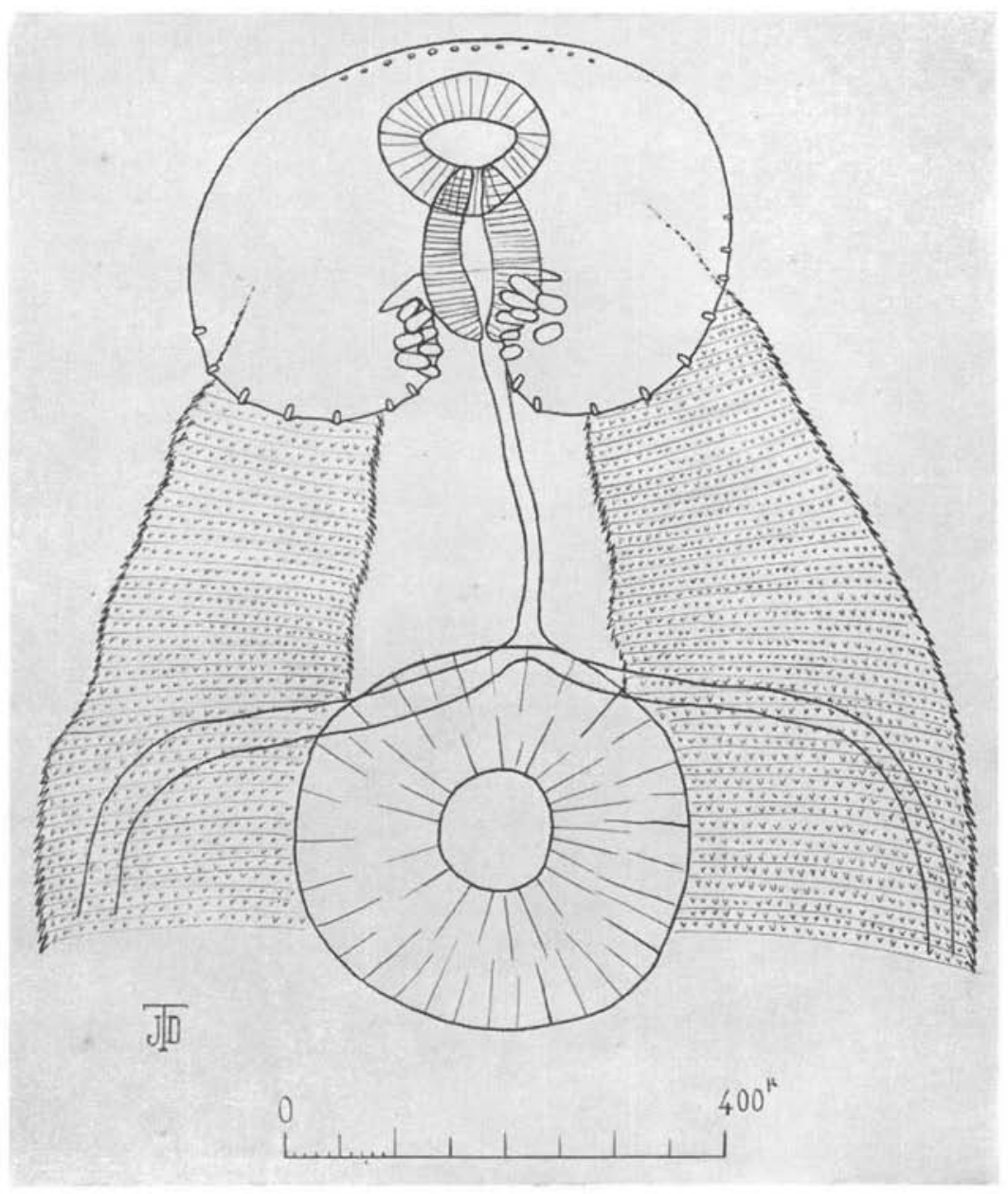

Fı́. 2. - Aporchis massiliensis nov. sp. Extrén:ité antérieure

$1,11 \mathrm{~mm}$., largeur, 0,600 à $0,630 \mathrm{~mm}$.; postérieur : longueur, 1,18 à $1,23 \mathrm{~mm}$., largeur, 0,54 à $0,55 \mathrm{~mm}$. Le postérieur est presque toujours un peu plus long que l'antérieur. La poche du cirre, sans atteindre le développement extraordinaire qu'elle présente chez 
A. croaticus Stossich, mesure 1,3 à $2 \mathrm{~mm}$. (y compris la vésicule séminale); sa portion antérieure contourne l'acétabulum et se termine immédiatement en avant.

Ovaire sphérique ou ovoïde, situé un peu en avant du testicule antérieur ; son diamètre (ou son grand axe) mesure 350 à $450 \mu$. La glande de Mehlis (environ $500 \mu$ ) est située immédiatement en arrière de l'ovaire; à son pôle postérieur est accolé le réservoir

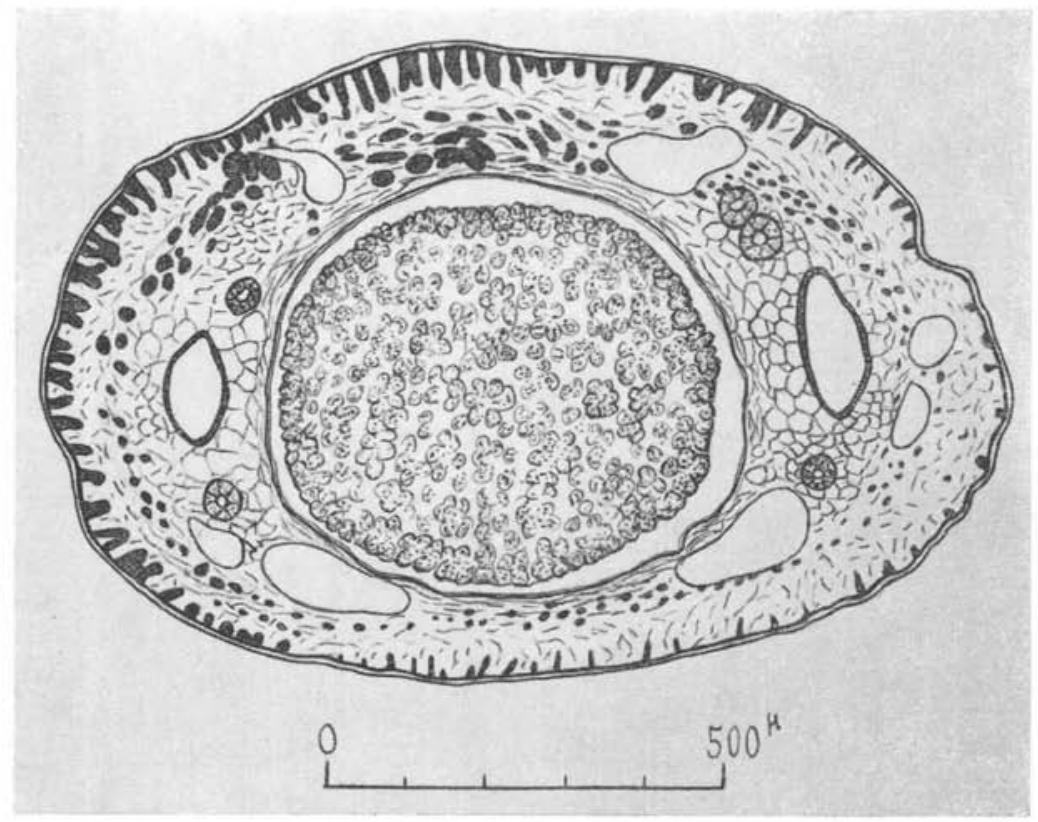

Fig. 3. - Aporchis massiliensis nov. sp. Coupe transversale au niveau du testicule antérieur. On remarquera la dissymétrie très accusée de la musculature longitudinale.

vitellin où aboutissent les deux vitelloductes transverses. Le trajet de ces canaux dessine une courbe très accusée, fortement concave en avant, contournant l'ovaire et la glande de Mehlis.

Les vitellogènes sont constitués par un grand nombre de follicules arrondis ou ovales ( 80 à $115 \mu$ ), alignés latéralement en dehors des branches intestinales ; leur limite antérieure se situe à peu près au milieu du corps, très loin en arrière de la vésicule séminale ; elle est nettement dépassée par la rampe utérine bourrée d'œufs. La limite postérieure correspond au niveau du bord antérieur ou du milieu de l'ovaire. 


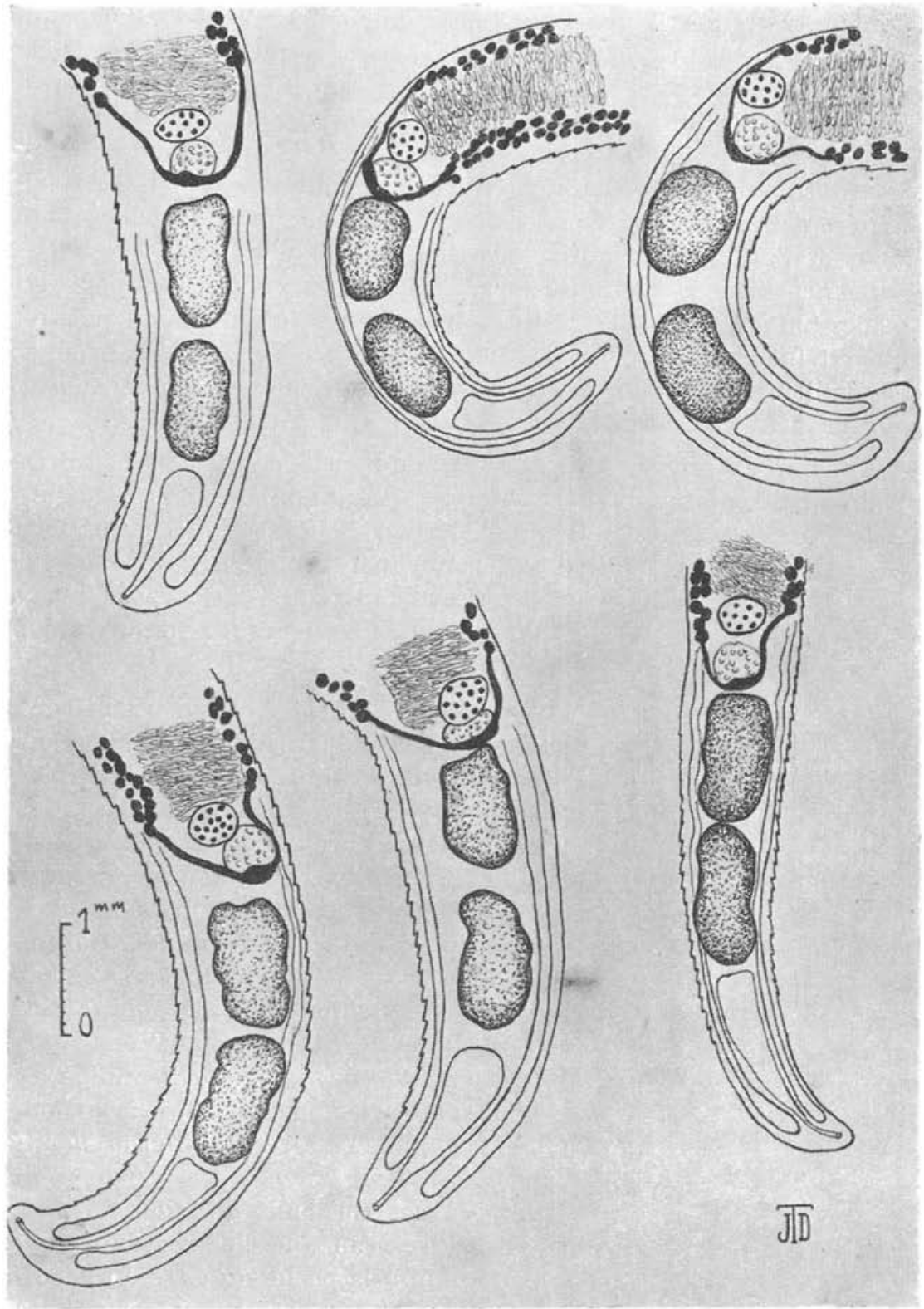

FIg. 4. - Aporchis massiliensis nov, sp Variations de l'extrémité postérieure chez six sujets 
La portion postérieure et moyenne de l'utérus constitue un tube dont le trajet décrit une ligne hélicoïdale depuis l'ootype jusqu'au milieu du corps. A l'origine, les tours de cette spire sont très serrés et peu apparents ; ils s'écartent progressivement vers l'avant. Dans toute cette portion, l'utérus renferme des œufs innombrables. Par contre, dans le tiers antérieur du corps, le tube utérin suit un trajet presque rectiligne et ne renferme jamais d'œufs; il est peu apparent dans cette région et vient déboucher près du bord antérieur de l'acétabulum après l'avoir contourné.

Les œufs ont la structure caractéristique chez A porchis : ils sont ovales (92 à $100 \mu \times 47$ à $49 \mu$ ), avec un très long filament polaire qui atteint environ $520 \mu$. La longueur exacte de ce prolongement est d'ailleurs difficile à mesurer en raison de leur enchevêtrement ; elle ne peut être évaluée que sur les derniers œufs isolés (fig. 5).

A ppareil excréteur : Une vessie volumineuse, de forme à peu près triangulaire, occupe l'espace intercæcal terminal, en arrière du tes-

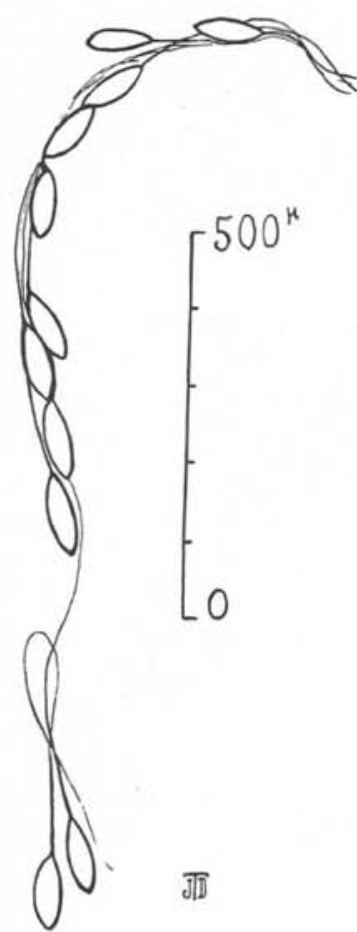

FIG. 5. - (Eufs d'Aporchis massiliensis nov. sp. ticule postérieur ; elle s'amincit en arrière et se termine par un canal qui débouche un peu avant l'extrémité du corps. Le trajet des canaux excréteurs n'a pu être suivi avec précision.

Discussion. - Les Aporchis sont des Echinostomatidæ, parasites intestinaux de Pélécaniformes et de Lariformes. Sur quatre espèces décrites jusqu'ici, une seule a été signalée en Europe : A. croaticus Stossich 1905, à Fiume, chez Carbo graculus (L.) (= Phalacrocorax aristotelis L.). Les trois autres sont: A. liouvillei Dollfus 1951, trouvé au Maroc (Mogador), chez Larus argentatus Pontopp., A. rugosus Linton 1928, aux Etats-Unis (Wood-Hole, Mass.), chez Sterna paradisea Brünnich, et A. segmentatus Fuhrmann 1915, à la Nouvelle-Calédonie (Yaté), chez Sterna bergii Licht.

L'espèce des Goélands de Riou ne peut être identifiée à aucune d'elles : chez croaticus $(39 \mathrm{~mm}$.), la poche du cirre est excessivement longue (un tiers du corps), les vitellogènes atteignent le niveau de la vésicule séminale, les testicules sont assez 
éloignés l'un de l'autre. Les spicules du plateau céphalique sont probablement au nombre de 31 .

Chez segmentatus $(21 \mathrm{~mm}$.$) ,$ les testicules sont à grand axe transversal, la poche du cirre mesure $1,3 \mathrm{~mm}$., les vitellogènes atteignent la vésicule séminale, le plateau céphalique compte 55 à 57 spicules et leur disposition est très différente. L'origine géographique est, de plus, très éloignée.

Chez liouvillei, la taille est petite $(7,2 \mathrm{~mm}$.), les testicules sont à grand axe transversal, les vitellogènes sont localisés dans le dernier tiers du corps. Les spicules caducs n'ont pas été vus.

A. rugosus $(17 \mathrm{~mm}$.$) me pa-$ rait être la forme la plus voisine de celle que je viens de décrire, mais les testicules sont à grand axe transversal et beaucoup plus petits (390 et $350 \mu)$; les spicules (caducs ?) n'ont pas été vus; la localisation géographique est très éloignée, l'hôte est différent.

Le tableau de la p. 454 résume ces caractères.

Fréquence et localisation. - J'ai trouvé Aporchis massiliensis quatre fois chez les Goélands de Riou (tous adultes). Les parasites étaient localisés entre le premier tiers et le milieu de l'intestin. Les dates sont les suivantes : 21 mai 1953 , un

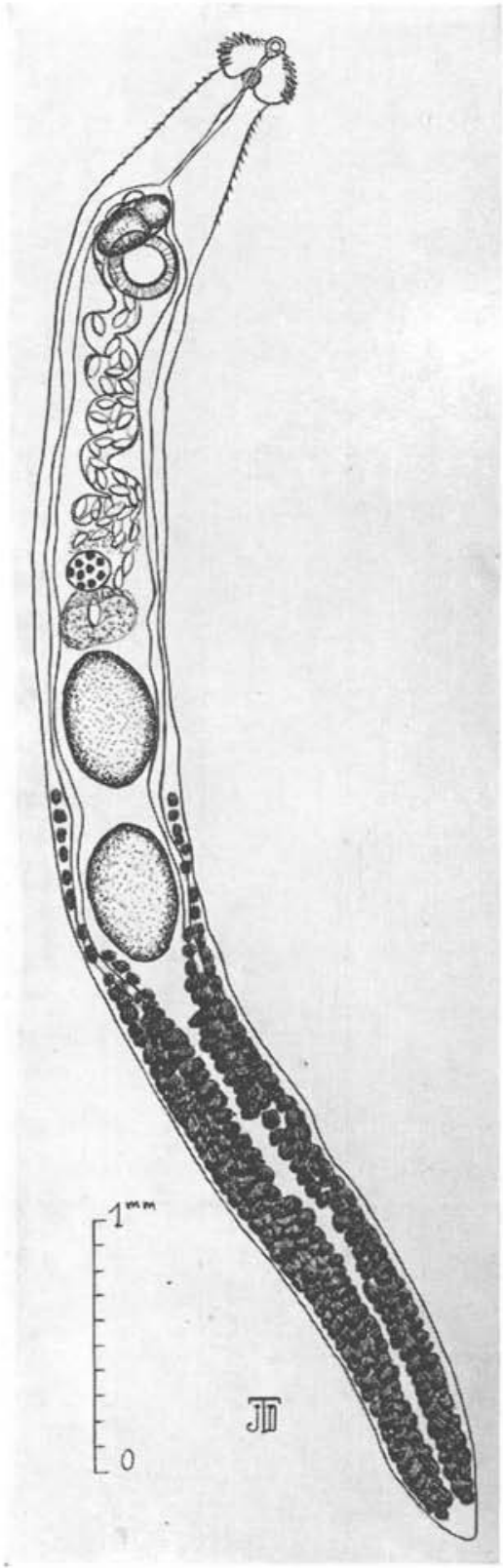

F1G. 6. - Mesorchis pseudoechinatus (Olsson 1876) 


\begin{tabular}{|c|c|c|c|c|c|c|c|c|}
\hline 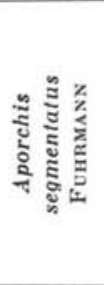 & 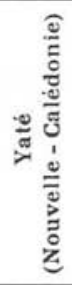 & 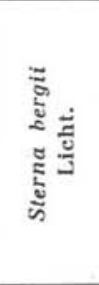 & $\begin{array}{l}\tilde{E} \\
\vec{a}\end{array}$ & 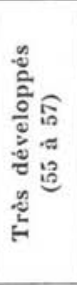 & 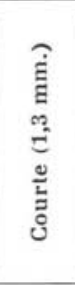 & 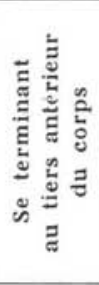 & 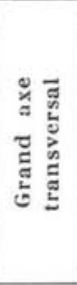 & 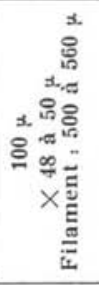 \\
\hline 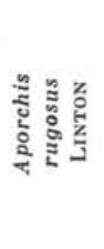 & 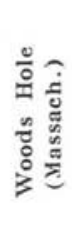 & 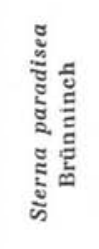 & $\begin{array}{l}E \\
E \\
E\end{array}$ & 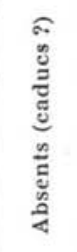 & 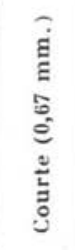 & 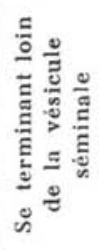 & 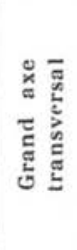 & 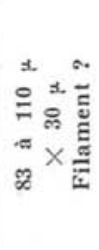 \\
\hline 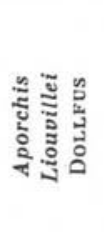 & 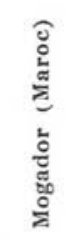 & 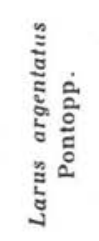 & $\begin{array}{l}\dot{\Xi} \\
\text { ㄱ } \\
\text { ํ. }\end{array}$ & 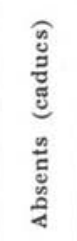 & 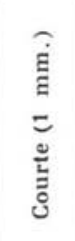 & 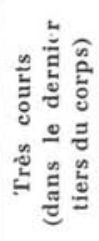 & 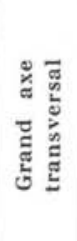 & 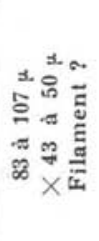 \\
\hline 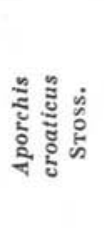 & 䓂 & 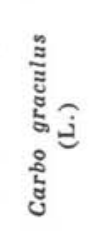 & $\begin{array}{l}\dot{\varepsilon} \\
\text { हี } \\
\stackrel{g}{\mathscr{g}}\end{array}$ & 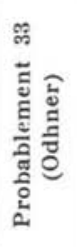 & 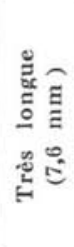 & 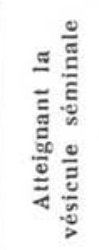 & 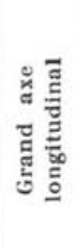 & 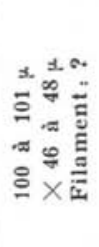 \\
\hline 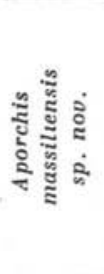 & 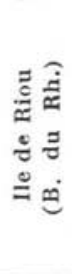 & 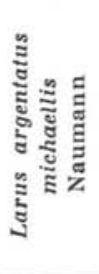 & 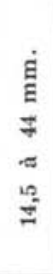 & 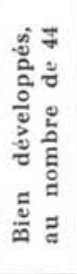 & 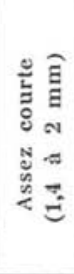 & 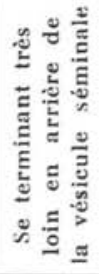 & 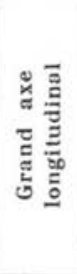 & 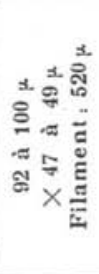 \\
\hline & 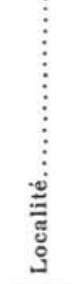 & $\begin{array}{l}\vdots \\
\vdots \\
\stackrel{0}{0}\end{array}$ & 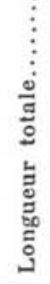 & 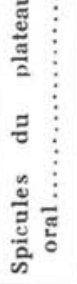 & 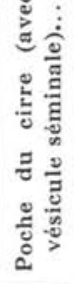 & 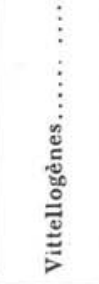 & 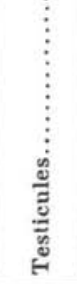 & $\dot{\Xi}$ \\
\hline
\end{tabular}


seul sujet; 11 mars 1954, un seul sujet; même jour, chez un autre Oiseau, six sujets ; 26 mars 1954, deux sujets. Au total, dix individus ont pu être examinés.

\section{MESORCHIS PSEUDOECHINATUS (Olsson 1876)}

Cet Echinostomatidé a été trouvé une seule fois à Riou (6 août 1953), chez un Goéland en plumage juvénile (environ 4 mois). 25 sujets ont été recueillis dans l'intestin, à $60 \mathrm{~cm}$. du pylore.

Il s'agit d'une espèce déjà signalée chez Larus argentatus et chez d'autres Oiseaux de mer en Scandinavie, en Europe Centrale, en Egypte, aux Etats-Unis. Edwin Linton l'a trouvée à Woods-Hole (Mass.), chez Larus argentatus, L. atricilla, L. delawarensis, L. marinus, L. philadelphia, Colymbus auritus, C. holbölli et Gavia immer.

Les exemplaires de Riou (fig. 6) sont allongés, à bords subparallèles ; ils mesurent 5,5 à $6,5 \mathrm{~mm}$. (moyenne $6 \mathrm{~mm}$.). Leur plus grande largeur, au niveau du testicule antérieur, varie de 0,41 à $0,47 \mathrm{~mm}$. (moyenne $0,43 \mathrm{~mm}$.). Le plateau céphalique, largement échancré ventralement, porte de chaque côté 11 spicules disposés sur un seul rang; les plus gros mesurent 52 à $54 \mu$ sur $12 \mu$. La largeur du plateau est comprise entre 275 et $320 \mu$ (moyenne $295 \mu$ ). Ventouse orale, 110 à $115 \mu$; acétabulum, 260 à $270 \mu$. Pharynx légèrement plus petit que la ventouse orale, long de 95 à $115 \mu$, large de $95 \mu$. L'ovaire est sphérique $(125$ à $155 \mu)$. Le testicule antérieur, situé un peu en avant du milieu du corps, mesure 475 à $560 \mu$ sur 260 à $320 \mu$; le postérieur, placé très près du premier, mesure 505 à $560 \mu$ sur 295 à $320 \mu$. Poche du cirre et vésicule séminale accolées en avant de l'acétabulum, dépassant un peu son diamètre. Les vitellogènes occupent la partie postérieure du corps et s'étendent jusqu'à l'espace intertesticulaire, parfois même jusqu'au tiers du testicule antérieur. CEufs : 78 à $82 \mu \times 47$ à $48 \mu$.

Ces mesures concordent de façon satisfaisante avec celles qui ont été données par Looss (1899, p. 685), par Dietz (1910, p. 460) et par Linton (1928, p. 13-14).

\section{Famille DICROCGELIDAE Odhner 1910}

BRACHYLECITHUM MICROTESTICULATUM nov. sp.

Parasite rare, trouvé une seule fois chez une $q$ adulte le 11 mars 1954 : trois sujets dans le premier tiers de l'intestin. Cette localisation peut paraître anormale, les Brachylecithum étant connus comme parasites 
des voies biliaires. Cependant, le foie examiné avec grand soin et dans sa totalité au binoculaire n'a fourni aucun autre exemplaire. Il faut reconnaître, d'ailleurs, que la littérature mentionne quelques autres trouvailles de Brachylecithum dans l'intestin, considérées comme anormales, par exemple : B. megastomum (Johnston 1917) chez Slerna bergii Licht., B. parvum (Johnston 1917) chez Streptera cuneicaudata (Vieill.), B. halcyonis (Yamaguti 1941) chez Halcyon coromanda maior (Temm. et Scheg.), etc.

Description. - Le corps est étroit et allongé (fig. 7), à bords parallèles ; longueur, 4,5 mm., largeur maxima, 0,39 mm. Ventouse orale, $195 \mu$. Acétabulum, 225 à $260 \mu$. Distance des deux ventouses centre à centre : $540 \mu$. Pharynx accolé à la ventouse orale $(90 \times$ $80 \mu)$. Esophage court $(50 \mu)$. La terminaison des branches intestinales masquée par les œufs n'a pu être observée. Deux testicules sphériques, situés l'un au-devant de l'autre, ne se touchant pas (intervalle $=110 \mu$ ), le premier plus petit $(135 \mu$ ), le second mesurant $165 \mu$; ils ne sont pas rigoureusement alignés dans l'axe. La poche du cirre ovoïde $(200 \mu)$ renferme une vésicule séminale sinueuse ; elle est située immédiatement en avant de l'acétabulum. L'ovaire est sphérique (diamètre $=180 \mu$ ), placé à $180 \mu$ en arrière du testicule postérieur. Glande de Mehlis $(60 \mu)$ accolée en arrière de l'ovaire. Les vitellogènes atteignent en avant le niveau de l'ovaire; ils sont constitués par un petit nombre de follicules de grande taille $(120$ à $180 \mu), 5$ à 7 de chaque côté ; leur extension correspond à $1 / 5,6$ de la longueur totale du corps. L'utérus remplit entièrement la région postérieure ; ses sinuosités passent entre les deux testicules, aboutissant à un métraterme accolé à la poche du cirre. Les œufs mesurent 36 à $38 \mu \times 20$ à $24 \mu$.

Discussion. - Les Dicrocœliidés ne paraissent pas fréquents chez les Lariformes: en dehors de Brachylecithum megastomum Johnston, déjà cité dans l'intestin de Sterna bergii Licht., en Australie, on peut mentionner Orthorchis lari Travassos, dans les canaux pancréatiques de Larus dominicanus Licht., au Brésil.

L'espèce que je décris ici paraît bien devoir être rangée dans le genre Brachylecithum, tel qu'il a été récemment défini à nouveau par R.-Ph. Dollfus (1954, p. 590 et p. 595, fig. K) : corps très allongé, à bords parallèles, sans élargissement au niveau de l'acétabulum et des testicules; ces derniers entièrement postacétabulaires (toutefois pas rigoureusement alignés dans l'axe du corps). Vitellogènes formés d'un petit nombre de follicules de grandes dimensions. 
Ce n'est pas sans quelques hésitations que je propose un nouveau nom spécifique pour un genre dont la systématique est déjà surchargée. Cependant, le Brachylecithum des Goélands de Riou ne semble pouvoir être assimilé à aucune des formes décrites.

Le caractère le plus remarquable réside dans la petite taille des testicules, tous deux (surtout le premier) plus petits que l'ovaire. Cette disposition est rare chez Brachylecithum: on l'observe chez B. baskakowi (Iwanitzky 1927) de Muscicapa grisoia L. (U.R.S.S.), chez B. kakea (Bhalerao 1926) de Corvus insolens (Indochine), chez $B$. kirghisensis Evronova 1952 de Montfringilla alpicola prosvirowi Zar. (U.R.S.S.), chez B. strigosum Looss 1899 de Merops apiaster L. (Egypte), et chez B. stunkardi (Pande 1939) de Garrulus lanceolatus (Inde). Toutes ces espèces diffèrent de celle de Riou, non seulement par leurs hôtes et leur provenance géographique, mais aussi par leur anatomie: chez $B$. baskakowi, les follicules vitellogènes sont au nombre d'une douzaine de chaque côté et occupent près du tiers du corps; chez B. kakea, les testicules et l'ovaire se touchent, la

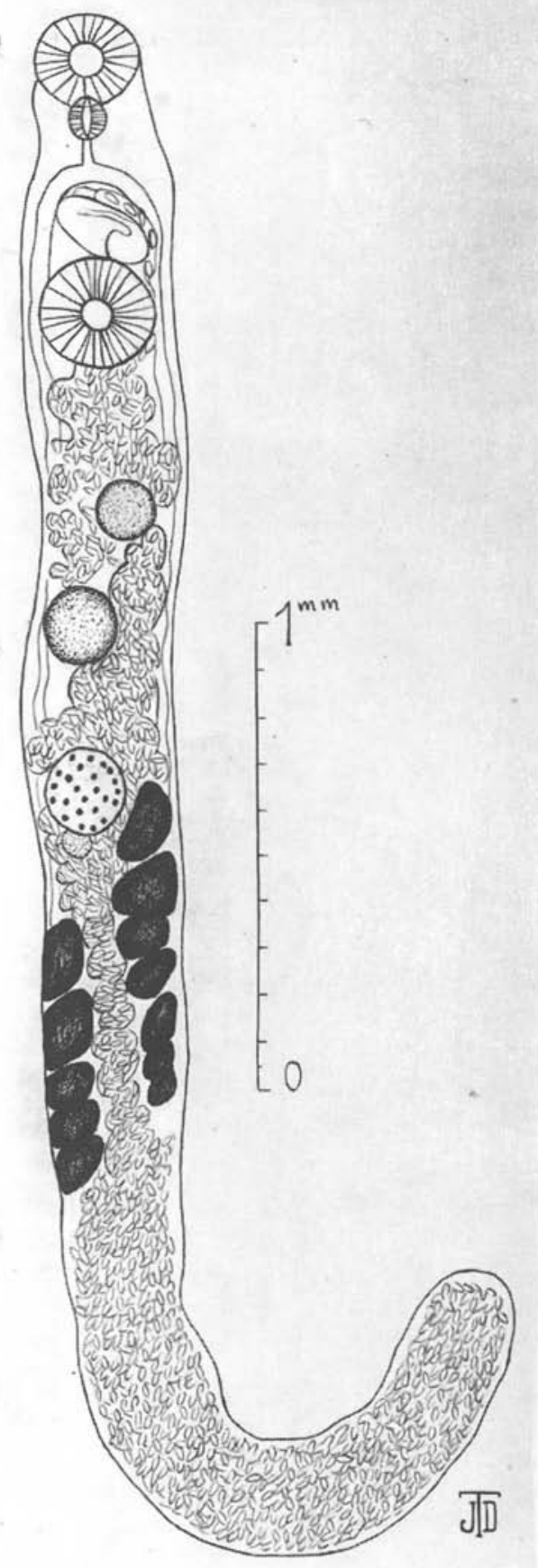

Fic. 7. - Brachylecithum microtesticulatun: nov. sp. 


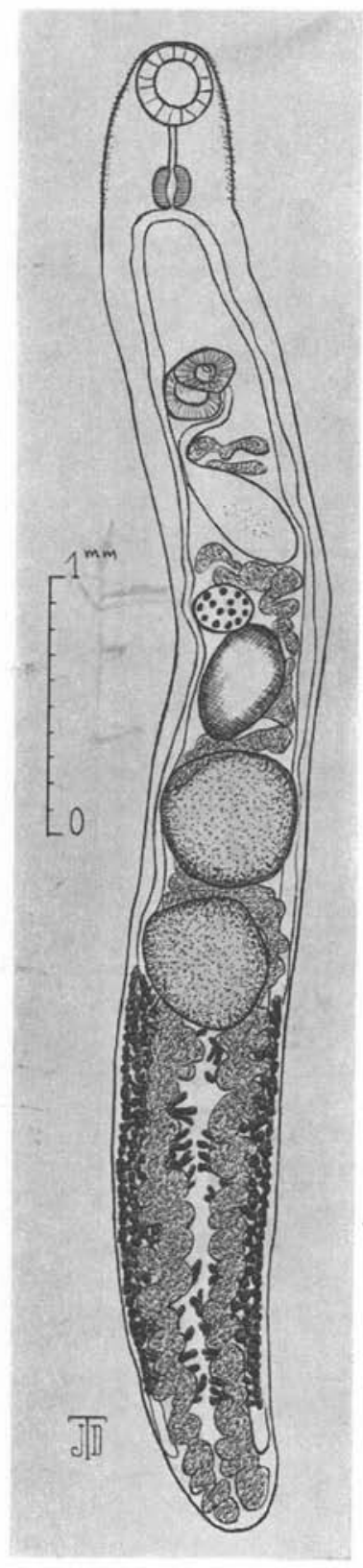

FIG. 8. - Knipowitschetrema echinatum nov. sp. poche du cirre est éloignée en avant de l'acétabulum; chez B. kirghisensis, le corps est beaucoup plus étroit et allongé, les testicules se touchent, la poche du cirre est petite; chez B. strigosum, les follicules vitellogènes sont plus petits et plus nombreux (jusqu'à 14 d'un côté), la poche du cirre très allongée dans l'axe du corps ; chez B. stunkardi, il y a douze à treize follicules vitellogènes de chaque côté, et leur début est bien en arrière de l'ovaire; les gonades se touchent presque.

\section{Famille GaLACTOSOMATIDAE Morosov 1950}

\section{KNIPOWITSCHETREMA ECHINATUM nov. sp.}

Description. - Corps allongé, linéaire, à bords subparallèles (fig. 8). Longueur totale : 4,6 à $7 \mathrm{~mm}$. (moyenne calculée sur 18 sujets : $5,1 \mathrm{~mm}$.). La plus grande largeur est comprise entre 0,49 et $9,76 \mathrm{~mm}$. (moyenne sur $18: 0,59$ ). Le rapport $\frac{\text { longueur }}{\text { largeur }}$ est donc égal à 8,6 . La cuticule est couverte d'épines nombreuses et serrées (fig. 9), particulièrement dans la région antérieure où leur longueur atteint $8 \mu$.

Ventouse orale terminale : diamètre, $300 \mu$. Acétabulum accolé à la ventouse génitale qui le recouvre en partie, formant un complexe situé un peu en avant du quart antérieur du corps. L'acétabulum mesure $220 \mu$ et la ventouse génitale $230 \mu$. La paroi du corps comprend une cuticule dont l'épaisseur est en moyenne de $4 \mu$, tapissée d'un revêtement de très nombreuses cellules glan- 
dulaires. Des faisceaux de fibres musculaires longitudinales, épais de 6 à $7 \mu$, forment une couche interne à peu près ininterrompue.

Tube digestif : Pharynx volumineux, un peu plus long que large $(180 \times 160 \mu)$, relié à la bouche par un long prépharynx (180 à $240 \mu$ ). (Esophage nul : la bifurcation a lieu dès le bord postérieur du pharynx ; les deux branches divergent immédiatement vers les

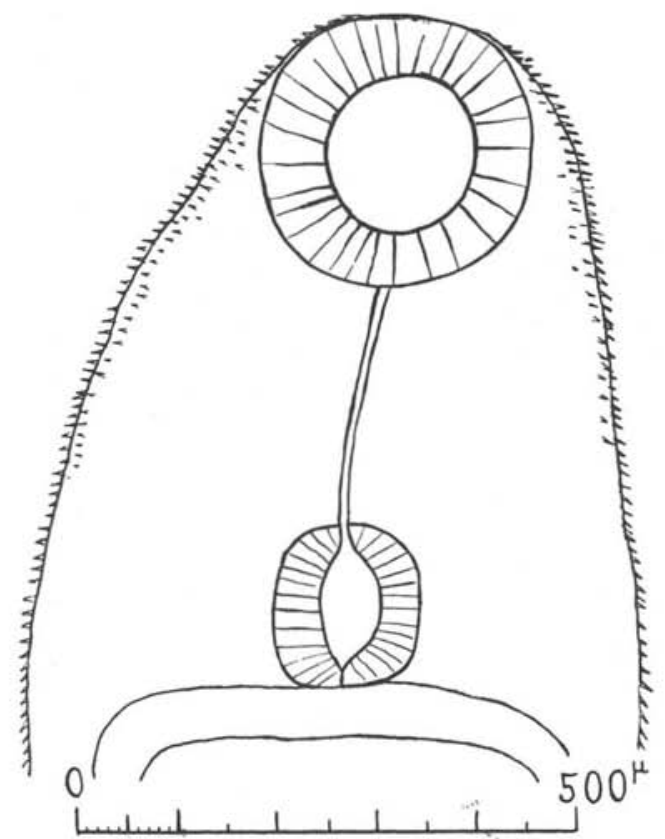

Fıg. 9. - Knipowitschetrema echinatum nov. sp. Extrémité antérieure montrant le développement des épines cuticulaires.

côtés du corps, puis se portent parallèlement en arrière et se terminent à environ $300 \mu$ de l'extrémité. Les coupes transversales montrent que la paroi du cæcum est constituée par un épithélium cylindrique à hautes cellules ( $12 \mu$ environ).

Organes génitaux: Testicules arrondis, volumineux et rapprochés, disposés l'un au-devant de l'autre ; l'antérieur est presqu'au milieu du corps; il est un peu plus grand que le suivant : 495 à $570 \mu$ pour le premier, 480 à $525 \mu$ pour le second. La vésicule séminale, très grande, est située entre l'ovaire et le complexe acétabulogénital ; sa partie postérieure, dilatée en ampoule $(500 \times 230 \mu)$, 
se prolonge antérieurement en un tube sinueux qui décrit un $\mathrm{S}$ en contournant l'acétabulum pour aboutir au sinus génital. Il n'y a pas de poche du cirre.

L'ovaire est arrondi ou faiblement elliptique $(220 \mu)$. Il existe un réceptacle séminal très développé, ovoïde $(460 \times 280 \mu)$, situé entre l'ovaire et le testicule antérieur. Les vitellogènes occupent la région comprise entre le niveau du milieu ou du tiers du testicule postérieur jusqu'à environ $500 \mu$ de l'extrémité du corps ; leur extension est un peu moindre que celle des cæcums qui les dépassent d'environ $200 \mu$. Ces follicules sont développés surtout sur les côtés et n'occupent pas la ligne médiane.

Les anses utérines se portent d'abord en arrière jusqu'à l'extrémité du corps, puis reviennent en avant en passant entre les deux testicules, contournent le réceptacle séminal et l'ovaire, et accompagnent la vésicule séminale pour aboutir au sinus génital. Les œufs mesurent 28 à $29 \mu$ sur 14 à $15 \mu$.

Fréquence et localisation. - Rectum de Larus argentatus michaellis Naum. à Riou. J'ai constaté que ce parasite est particulièrement abondant chez les jeunes Oiseaux (un mois et demi à deux mois), qui l'hébergent dans la proportion de $24 \%$ : jusqu'à 118 sujets chez un même hôte ; il est beaucoup plus rare chez les adultes (trouvé une seule fois). II est vraisemblable qu'une certaine immunité doit jouer dans ce dernier cas.

Discussion. - Le genre Knipowitschetrema a été créé par Issaitschikov (1), en 1927, avec l'espèce $K$. nicolä̈, découverte en U.R.S.S., dans l'intestin de Larus argentatus Pont. Morosov (1950) a proposé la sous-famille des Knipowitschetrematinæ, groupant les genres Knipowitschetrema Issaitschikov, Ponticotrema Issait. et Tauridiana Issait. dans la famille des Galactosomatidx.

L'espèce que je viens de décrire est évidemment très voisine de $K$. nicolaï, mais je pense qu'elle doit être considérée comme distincte pour les raisons suivantes :

$1^{\circ}$ La cuticule porte un revêtement d'épines très développées, surtout dans la région antérieure ; elle est lisse chez nicolaï.

$2^{\circ}$ La forme générale du corps est beaucoup plus allongée; le rapport $\frac{\text { longueur }}{\text { largeur }}$ atteint ici 8,6 en moyenne (jusqu'à 9,9 chez certains sujets) et seulement 6,2 à 6,8 chez $K$. nicolaï.

(1) Il m'a été impossible de me procurer le travail original d'Issaitschikov, publié dans le "Festschrift für Knipowitsch », et je ne le connais que par la reproduction détaillée qu'en donne K. I. Skrjabin dans son traité (T. VI, p. 460464). C'est d'après ce dernier auteur que sont donnés les chiffres discutés ici. 
$3^{\circ}$ La position du complexe acétabulo-génital est nettement plus antérieure : en avant du quart, tandis qu'elle correspond exactement au tiers du corps chez nicolaï.

$4^{\circ}$ La taille est beaucoup plus grande : près du double.

$5^{\circ}$ La localisation dans le tube digestif de l'hôte est différente : j'ai toujours trouvé mes exemplaires dans le rectum, à quelques centimètres seulement de l'orifice anal ; Issaitschikov précise que son espèce a été trouvée dans l'intestin moyen.

$6^{\circ}$ L'hôte est le même, mais la distribution géographique est différente; aucune espèce de Knipowitschetrema n'avait été signalée jusqu'ici en France.

J'ajoute que le Trématode que j'avais signalé à Riou dans ma note préliminaire de 1935 , sous le nom de Microlistrum cochlear Dies., d'après du matériel macéré, insuffisamment fixé, appartient certainement à l'espèce décrite ici et doit être

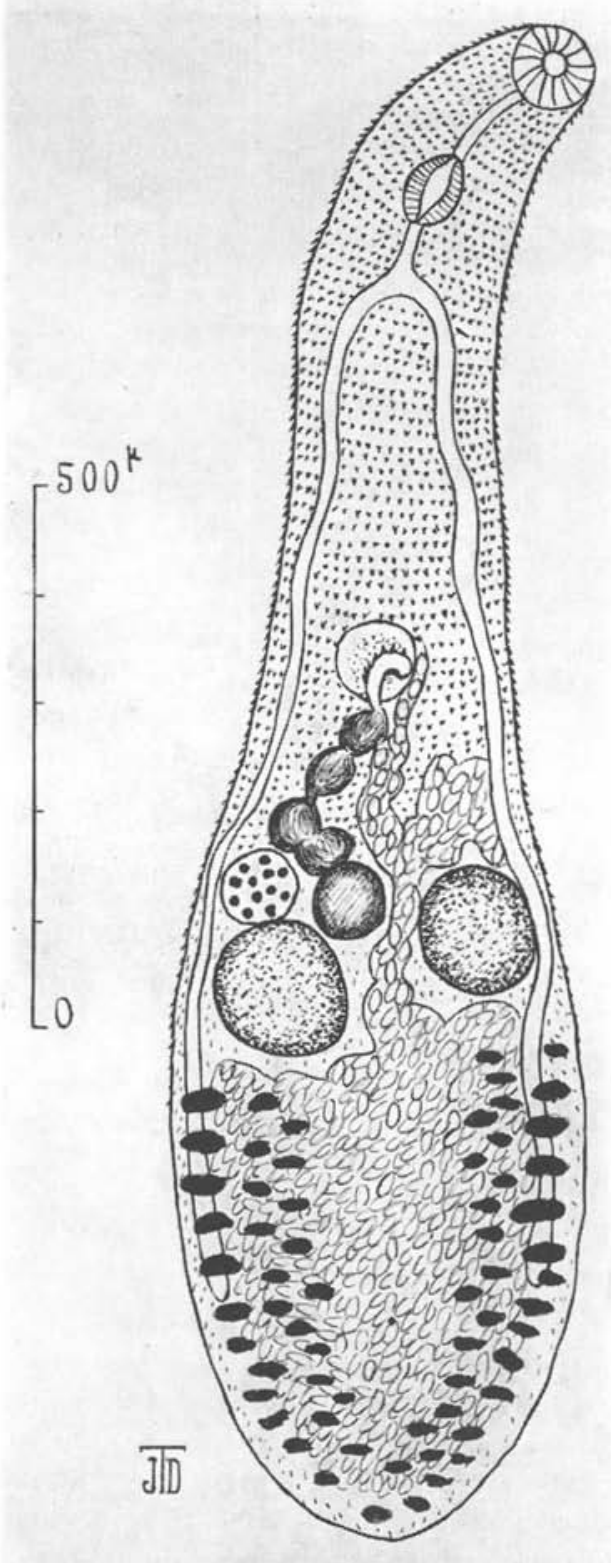

FiG. 10. - Stictodora sawakinensis Looss, 1899 rayé de la liste des parasites de L. argentatus dans cette localité. 
Gycle évalutif. - D'après Issaitschikov, les mètacercaires de Knipowitschetrema seraient enkystées dans les feuillets branchiaux de Belone acus Risso. Je n'ai pas d'observations personnelles à apporter sur cette question.

\section{STICTODORA SAWAKINENSIS Looss 1899}

Ce Trématode peut échapper facilement à l'attention en raison de sa très petite taille (fig. 10). Je l'ai trouvé cinq fois, toujours chez des Goélands adultes, localisé dans le rectum ou dans le dernier tiers de l'inlestin ; le nombre, chez chaque hôte, variait de 4 à 40 (le plus souvent 25 à 30). Dates des dissections : 27 mars 1953 ; 12 novembre 1953 ; 20 mars 1953 (deux oiseaux parasités) ; 5 avril 1955.

C'est un parasite classique des Larus et Puffinus qui peut, à l'occasion, se développer chez un Mammifère (chien en particulier), comme c'est le cas pour beaucoup de représentants de la superfamille des Heterophyoidea. Les caractères des sujets que j'ai recueillis (fig. 10) concordent de façon satisfaisante avec la description de Looss. La découverte de $S$. sawakinensis chez les Goélands du littoral de la Provence étend sensiblement l'aire de cette espèce connue notamment en Egypte, en Tunisie, à Suez et en Palestine.

\section{Famille HETEROPHYIDAE Odhner 1914}

\section{CRYPTOCOTYLE JEJUNA Nicoll 1907}

Ce parasite est fréquent à Riou chez les jeunes I.arus âgés de 45 à 75 jours $(30 \%)$, beaucoup moins chez les adultes. On le trouve dans toute la longueur de l'intestin, souvent en grand nombre (plusieurs centaines, parfois plus d'un millier).

Je crois inutile de redonner une description de cette espèce souvent citée (voir Ransom, 1920 ; Nicoll, 1923 ; Ciurea, 1933 ; Witenberg, 1929 ; Price, 1931 ; Rothschild, 1938 ; Skrjabin, 1952). Je me bornerai à indiquer que les sujets examinés sont longs de 1,7 à $1,8 \mathrm{~mm}$. et larges de 0,65 à $0,7 \mathrm{~mm}$. Les œufs mesurent en moyenne $32 \times 16 \mu$; le diamètre du sinus génital ne dépasse pas $95 \mu$; les vitellogènes n'atteignent pas le milieu de la distance qui sépare le sinus génital de la bifurcation du tube digestif. Ces caractères éliminent sans hésitation $C$. lingua (Creplin 1825), dont les œufs sont beaucoup plus gros $(47 \times 22 \mu$ en moyenne) et le sinus génital beaucoup plus grand (voir redescription de cette espèce dans le travail de R.-Ph. Dollfus, 1952, p. 139-149). 
Dans ma note préliminaire de 1935, j'avais cru pouvoir rapporter le Cryptocotyle de Riou à C. concavum (Creplin 1925). Je pense maintenant que cette attribution était inexacte : la forme générale moins allongée et la disposition des testicules qui ne sont pas sur une même ligne horizontale éliminent $C$. concavum.

\title{
Famille MICROPHALLIDAE Viana, 1924
}

\author{
GYMNOPHALLUS DELICIOSUS (Olsson 1893), Odhner 1900
}

Ce parasite a été trouvé deux fois chez les Goélands de Riou : une première fois (26 mars 1954), deux sujets chez une + adulte ; une seconde fois (5 avril 1955) six, également chez une $q$ adulte, dans la vésicule biliaire. Les caractères sont conformes à la description d'Odhner pour Gymnophallus deliciosus (Olss.).

Voici quelques mesures relevées sur mes exemplaires (fig. 11) : longueur totale, 1,9 à $2,2 \mathrm{~mm}$. ; largeur maxima, 0,7 à $0,8 \mathrm{~mm}$. Ventouse orale, 220 à $260 \mu$; acétabulum, 190 à $220 \mu$; pharynx, $50 \times$ $60 \mu$; œsophage, 120 à $130 \mu$; cæcums courts, ne dépassant pas le bord antérieur des testicules, ces derniers très volumineux (long., 500 à $550 \mu$; larg., 300 à $380 \mu)$. Ovaire sphérique $(170 \mu)$. Les vitellogènes sont formés de follicules de grande taille $(100$ à $170 \mu)$, en petit nombre ( 6 à 7 de chaque côté), groupés en deux amas de part et d'autre de l'acétabulum. Les anses utérines occupent toute la partie postérieure du corps et se prolongent par une boucle antérieure qui atteint le niveau de la bifurcation du tube digestif. Les œufs mesurent $23 \times 13,5 \mu$. Cuticule couverte de fines épines.

\section{Famille PACHYTREMATIDAE Baer 1943}

\section{PACHYTREMA CALCULUS Looss 1907}

Le genre Pachytrema Looss 1907 (= Minuthorchis Linton $1928=$ Multivitellaria Phadke et Gulati 1929) groupe une série d'espèces (Skrjabin en mentionne 8 dans son traité, t. IV, 1950), parasites de la vésicule hiliaire des Lariformes et aussi, plus rarement, des Charadriformes et de certains Passériformes (Corvidés). Il ne paraît pas avoir jamais été signalé en France. Deux espèces sont connues en Europe : P. calculus Looss 1907 en Italie (Trieste), chez Larus argentatus et L. ridibundus, indiqué aussi par Odhner dans la vésicule biliaire de $L$. fuscus à l'ile de Väderöarna (Suède) et $P$. paniceum Brinkmann 1942, chez L. fuscus à Herdla, près Bergen (Norvège). 


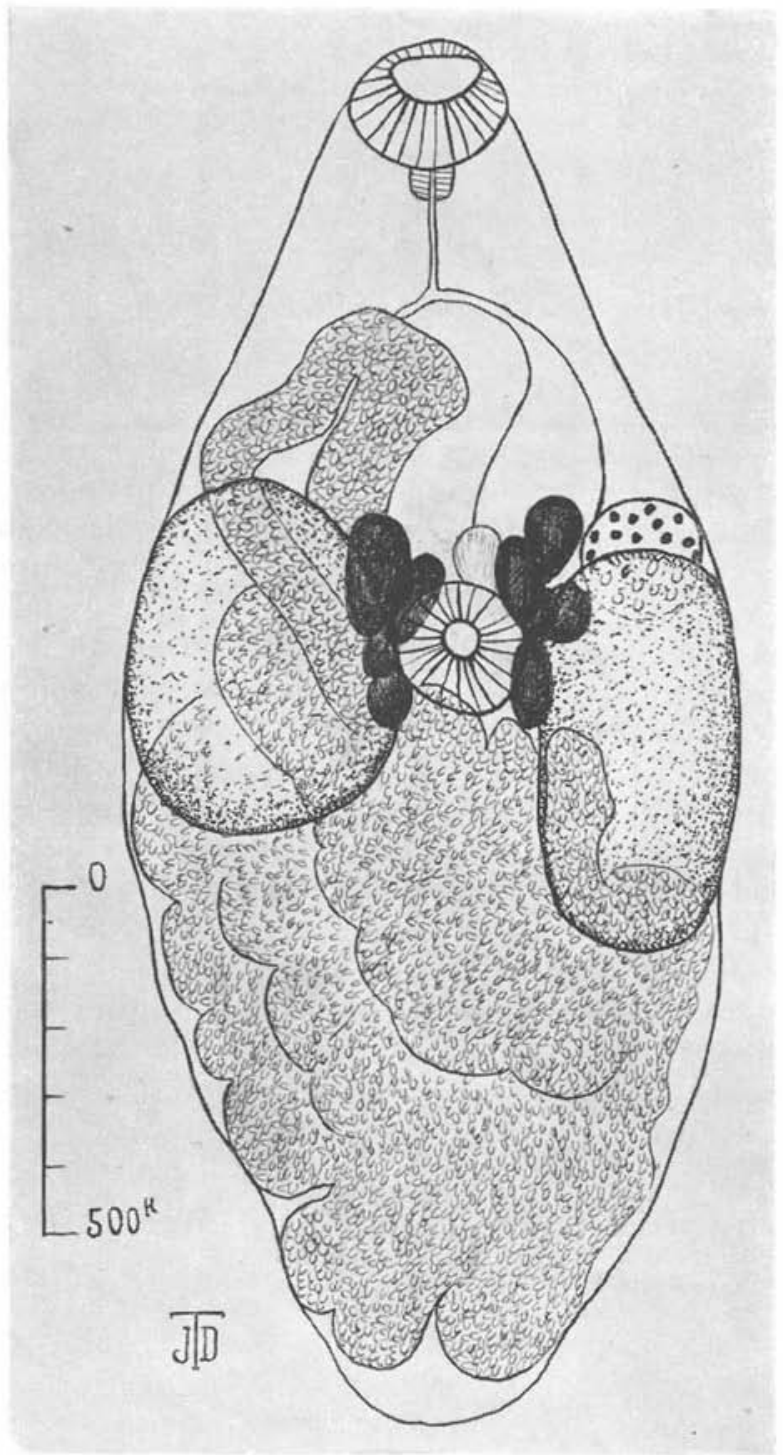

FiG. 11. - Gymnophallus deliciosus (Olsson, 1893)

Ces parasites sont considérés comme très rares : j’ai cependant trouvé trois fois des Pachytrema dans la vésicule des Goélands de Riou : une première fois (31 mai 1952), un sujet unique et immature chez un jeune Oiseau d'environ deux mois; une seconde fois (7 mars 1953), deux 
sujets complètement développés, qui remplissaient entièrement la vésicule de l'hôte fortement dilatée (un $\delta$ adulte). Une troisième fois (5 avril 1955), un sujet unique bien développé (chez un $\delta$ adulte). Tous ces Pachytrema avaient une coloration verdâtre due, évidemment, aux pigments biliaires.

Chez les individus complètement développés, le corps est orbiculaire, aplati dorsalement et fortement convexe du côté ventral. A l'état de contraction, les dimensions ne dépassent pas $8 \times 7 \mathrm{~mm}$. Chez les sujets en extension et légèrement comprimés (fig. 12), la longueur atteint 11,5 à 12,5 mm., la largeur 8,2 à $8,6 \mathrm{~mm}$. Les deux ventouses sont très rapprochées l'une de l'autre : la distance de centre à centre varie de 0,6 à $1,3 \mathrm{~mm}$. Le grand axe de la ventouse orale (transversal) mesure 465 à $480 \mu$; celui de l'acétabulum, 465 à $480 \mu$. Il n'y a pas de prépharynx ; le pharynx est aussi long que large $(195 \mu)$; l'œsophage nul ou excessivement court. Les deux branches intestinales se prolongent très loin en arrière et se terminent côte à côte à environ $1 \mathrm{~mm}$. de l'extrémité postérieure du corps. Le pore génital médian s'ouvre entre l'acétabulum et la bifurcation du tube digestif. Les deux testicules sont relativement très petits, ovoïdes $(500 \mu$ dans le sens transversal) et disposés symétriquement de chaque còté, vers le cinquième postérieur du corps. Le canal déférent remonte suivant l'axe médian et aboutit à une vésicule séminale située immédiatement en avant de l'acétabulum. L'ovaire $(475 \mu)$ est médian, légèrement en arrière d'une ligne transversale qui réunirait les testicules; la glande de Mehlis est accolée. Les follicules vitellogènes sont disposés en grappes bien distinctes, formant une sorte de couronne largement interrompue en avant et très faiblement en arrière. Les dernières grappes ont tendance à se fusionner ; leur nombre total varie de 22 à 25 de chaque côté ; chaque grappe mesure de 500 à $600 \mu$; leur limite antérieure n'atteint pas le niveau de l'acétabulum; leur limite postérieure dépasse l'extrémité du cæcum. Le vitelloducte longitudinal devient très apparent dans sa moitié postérieure et décrit une série d'arceaux très élégants, convexes en dedans, entre les grappes de follicules. Le vitelloducte transverse est à la hauteur de l'ovaire et aboutit à un réservoir vitellin. Les circonvolutions utérines occupent presque tout l'espace délimité par la couronne des vitellogènes; elles recouvrent presque entièrement les branches intestinales. Les œufs mesurent 114 à $116 \mu \times 52 \mu$.

Discussion. - Il convient d'abord d'éliminer divers Pachytrema manifestement très éloignés de l'espèce de Riou par leur anatomie, 


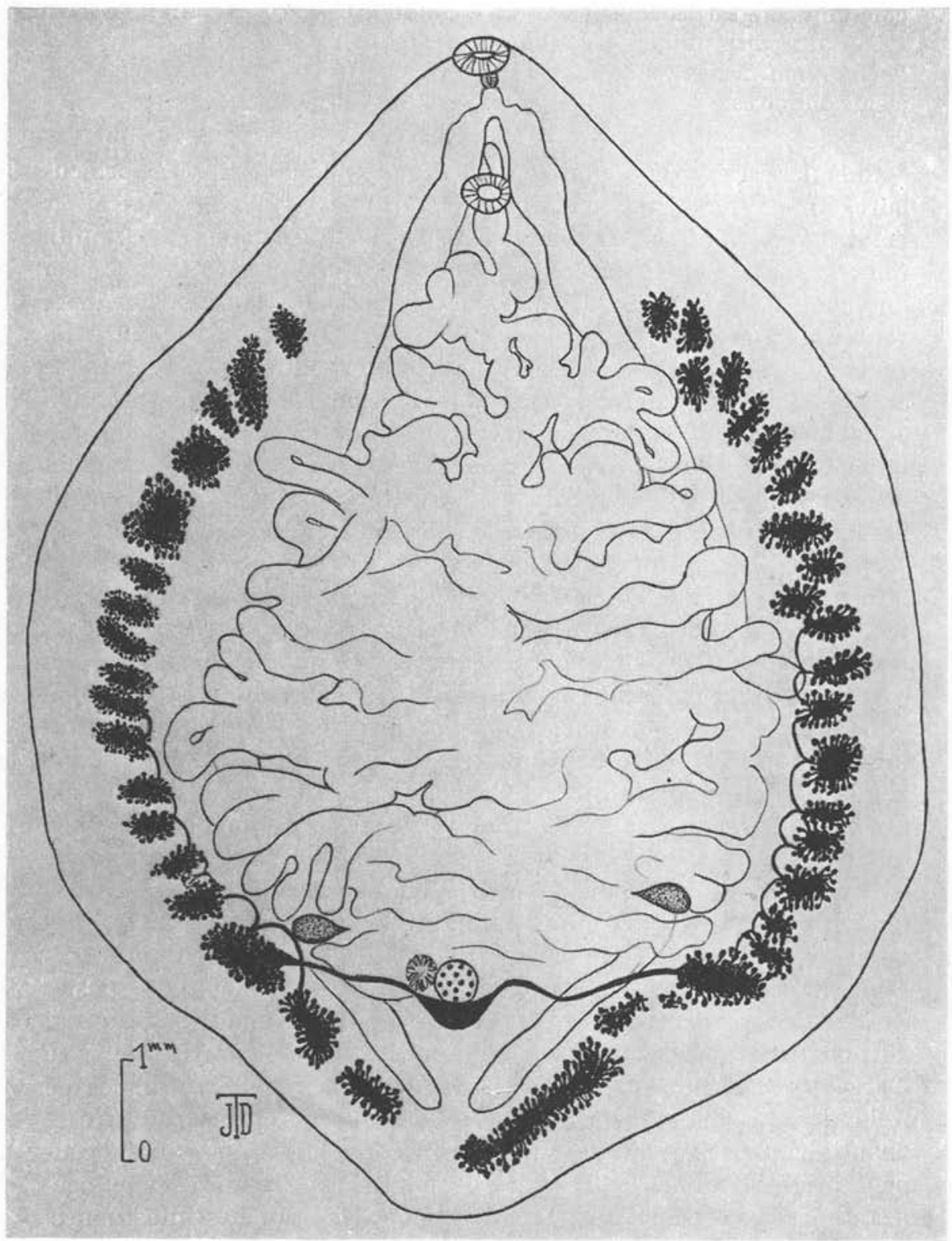

Fig. 12. - Pachytrema calculus Looss, 1907 Sujet légèrement comprimé sous lamelle 
leur hôte et leur distribution géographique; ce sont : $P$. hewletti (Phadke et Gulati 1929) de Corvus insolens (Inde); P. proximum Travassos 1921 d'Heteropygia fuscicollis (Brésil) ; P. tringæ Layman 1926 de Tringa alpina (U.R.S.S.).

Parmi les Pachytrema de Lariformes: P. magnum Travassos 1921 de Sterna magna (Brésil) est très différent par sa forme générale ; $P$. compositum Tscherbowitsch 1946 de Sterna longipennis (U.R.S.S.) a des œufs beaucoup plus petits $(82 \mu)$; $P$. sanguineum (Linton 1928) Purvis 1937 de Larus atricilla (U.S.A.) a été considéré par Odhner (1928) comme synonyme de $P$. calculus et par Pereira (1928) comme identique à $P$. magnum. La discussion ne peut donc porter que sur les deux espèces européennes: $P$. paniceum Brinkmann (1942) et $P$. calculus Looss 1907. La première a été étudiée en détail et son anatomie est fort bien connue ; elle se distingue en particulier par la grande taille des testicules qui font saillie sous la cuticule, par l'existence d'un œsophage relativement long et par l'absence de réservoir vitellin. Tous ces caractères font défaut chez l'espèce de Riou, qui doit done être déterminée Pachytrema calculus Looss 1907.

\section{Famille PHILOPHTHALMIDAE Looss 1899}

PHILOPHTHALMU'S sp.

Un unique exemplaire de cet intéressant Distome a été trouvé le 21 mai 1953 dans le cul-de-sac conjonctival (œil gauche) d'un ơ adulte.

Description. - La cuticule porte de très petites écailles; le corps est ovale (fig. 13); sa longueur totale ne dépasse pas $2,6 \mathrm{~mm}$., sa largeur maxima $0,85 \mathrm{~mm}$. Ventouse orale, $360 \mu$. Acétabulum très robuste, $570 \mu$. Le rapport $\frac{\text { acétabulum }}{\text { vent. orale }}$ atteint donc 1,58. La distance des deux ventouses (centre à centre) mesure $930 \mu$. Le pharynx, très robuste, atteint presque la taille de la ventouse orale; il est sphérique $(300 \mu)$. Il n'y a ni prépharynx, ni œsophage. Les branches intestinales se prolongent très loin en arrière, dépassant le milieu du testicule postérieur. Les deux testicules, non lobés, sont accolés l'un à l'autre ; ils sont plus larges que longs $(300 \times$ $170 \mu$ et $300 \times 200 \mu$ ), situés dans le dernier cinquième du corps. Ovaire de forme ovoïde $(250 \times 200 \mu)$, à peu de distance en avant 


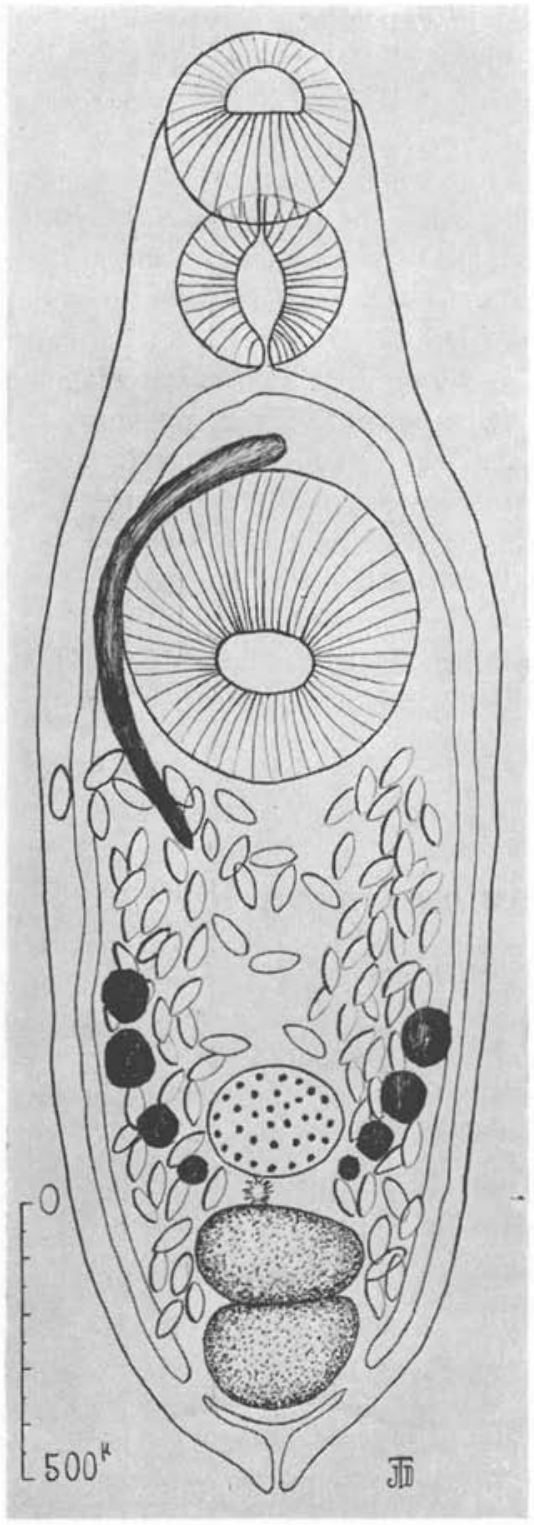

F1G. 13. - Philophihalmus sp. Sujet unique trouvé dans le cul-de-sac conjonctival. du testicule antérieur. Poche du cirre étroite et allongée, contournant l'acétabulum. Vitellogènes formés d'un petit nombre de follicules arrondis $(90$ à $100 \mu)$, décrivant une courbe qui entoure l'ovaire. Glande de Mehlis petite, entre l'ovaire et le testicule antérieur. Les anses utérines occupent une grande partie du corps en arrière de l'acétabulum ; elles se prolongent sur les côtés du testicule postérieur et recouvrent de chaque côté les vitellogènes et les cæcums. Les œufs sont grands : $92 \times 40 \mu$. La vessie petite, triangulaire, émet un tronc médian qui débouche à l'extrémité du corps et reçoit deux canaux antérieurs qui contournent le dernier testicule.

Discussion. - Il est possible que le Philophthalmus de Riou corresponde à une nouvelle espèce, mais je préfère laisser ce point provisoirement en suspens, dans l'attente de matériel plus abondant permettant d'observer une série d'individus. On a décrit plus d'une douzaine d'espèces de ce genre; parmi celles qui appartiennent aux Lariformes, $P$. lucipetus (Rudolphi), P. lacrymosus Braun et P. skrjabini Efimov ne correspondent pas au type de Riou. 


\section{Famille RENICOLIDAE R.-Ph. Dollfus 1939}

\section{RENICOLA LARI J. 'Timon-David 1933}

Je renvoie pour la description de cette espèce à mon travail de 1933 et à la révision du genre Renicola publiée par R.-Ph. Dollfus (1946, p. 60-61).

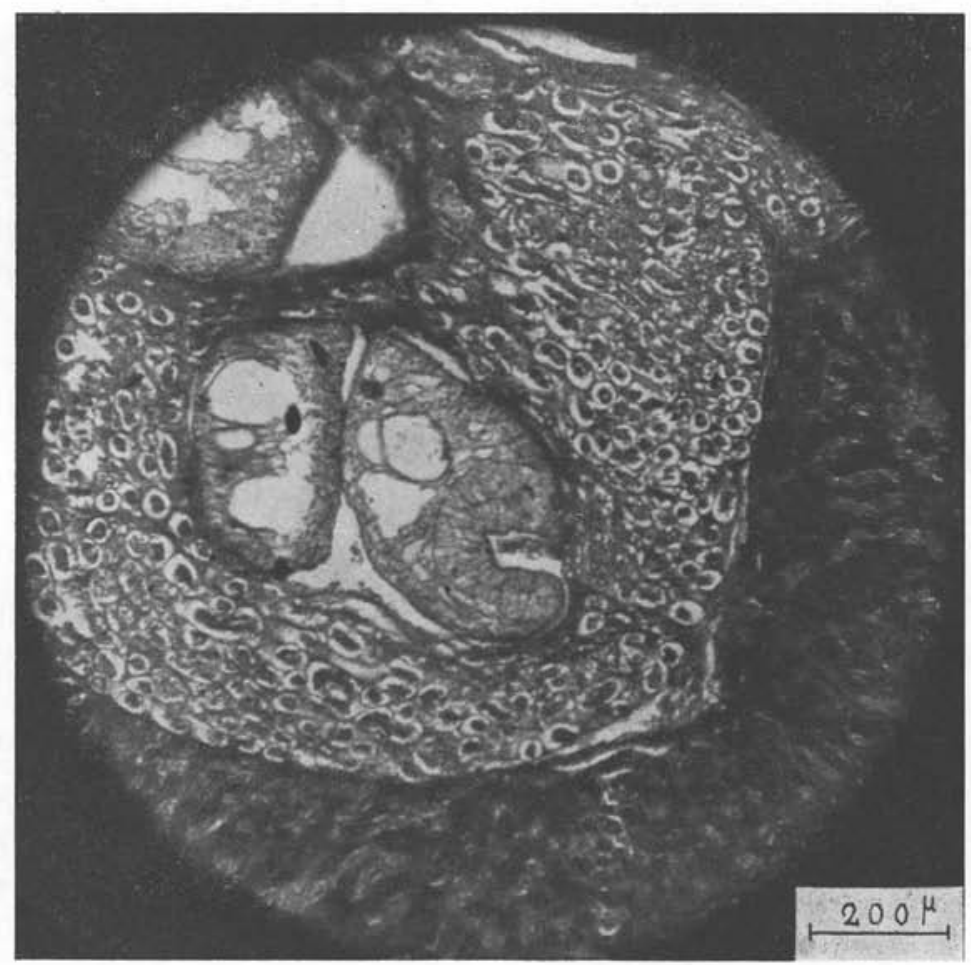

FIG. 14. - Rein de Larus argentatus michaellis avec Renicola lari T.D. On remarquera la localisation des parasites dans les tubes excréteurs : la zone glomérulaire est intacte.

Quand j'ai décrit $R$. lari, aucune espèce de ce genre n'avait été signalée en France et il était permis de penser qu'il s'agissait d'une grande rareté. Depuis cette époque, mes recherches à Riou m'ont permis de constater que $R$. lari est, au contraire, d'une extrême abondance dans cette localité (et vraisemblablement sur d'autres points du littoral méditerranéen). C'est - de loin - le Trématode le plus commun chez les 


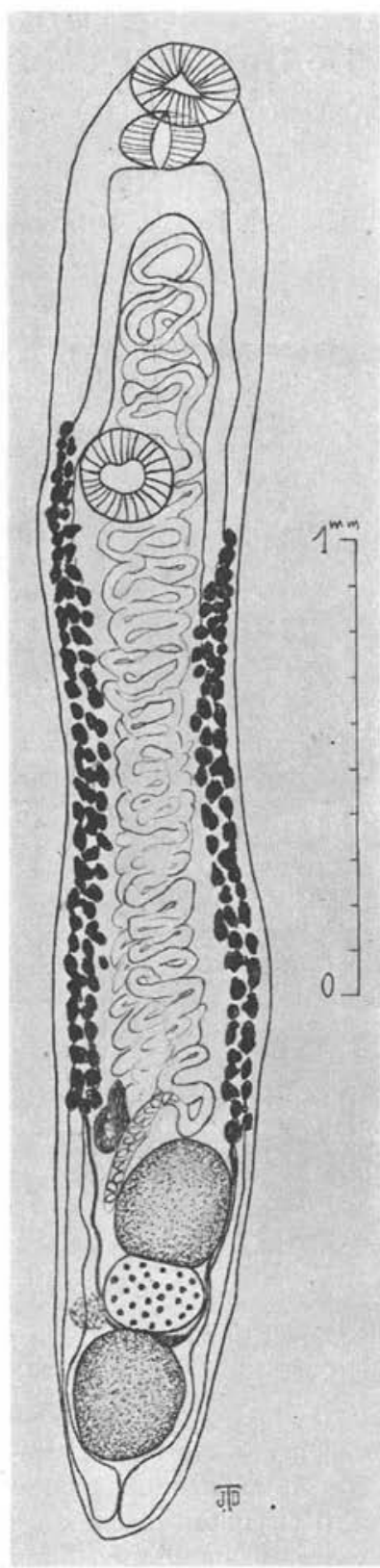

F1G. 15. - Brachylaemus sp.
Goélands de Riou : le nombre des sujets que j'ai eu sous les yeux se chiffre par centaines; la fréquence de l'infestation atteint $80 \%$ chez les adultes; elle est plus rare chez les jeunes de 2 à 3 mois (12\%). Cette notion d'épidémiologie présente une certaine importance car elle peut mettre sur la voie de la découverte du cycle évolutif. On sait que C. A. Wright (1953) a suggéré récemment que les Renicola des Oiseaux de mer pourraient correspondre à une cercaire du groupe Rhodometopa. Cette hypothèse est basée sur l'analogie de l'appareil excréteur.

La microphotographie (fig. 14) que je reproduis ici montre la localisation de Renicola lari dans les canaux urinifères d'un Goéland de Riou.

\section{Famille BRACHYLAEMIDAE Joyeux et Foley 1930}

\section{BRACHYLAEMUS sp.}

J'ai trouvé une seule fois un représentant du genre Brachylaemus chez un Goéland ( $q$ adulte) tué à Riou le 5 avril 1955. Cet exemplaire unique a été recueilli dans le dernier tiers de l'intestin. Etant donné l'extrême difficulté - pour ne pas dire l'impossibilité - d'attribuer un nom spécifique à un Brachylaemus isolé, je me borne à signaler sa présence sans préciser davantage. Il semble bien que ce Brachylaemus se trouve ici chez un hôte exceptionnel : dans sa monographie de 1934-35, R.-Ph. Dollfus ne cite aucune espèce de ce genre chez un Lariforme ; le traité de K. I. Skrjabin (t. II, 1948, p. 171-205) n'en fait également aucune mention.

Les dimensions sont les suivantes (fig. 15) : longueur totale, $3,5 \mathrm{~mm}$. ; largeur maxima, 0,44 mm. ; ventouse orale, 
$190 \mu \times 242 \mu$ (largeur) ; acétabulum, $207 \mu$; pharynx, $103 \mu \times$ $190 \mu$ (largeur) ; testicule antérieur, $294 \times 260 \mu$; testicule postérieur, $294 \times 277 \mu$; ovaire, $173 \times 225 \mu$; œufs, 27 à $28 \mu \times 14$ à $15 \mu$. Les vitellogènes atteignent en avant le niveau du bord antérieur de l'acétabulum ; en arrière, celui du bord antérieur du premier testicule. Les téguments sont dépourvus d'épines.

\section{Famille STRIGEIDAE Raillet 1919}

\section{CARDIOCEPHALUS LONGICOLLIS (Rudolphi 1819) Szidat 1928}

J'ai trouvé ce Strigéidé cinq fois à Riou, toujours dans le premier tiers de l'intestin, chez des Goélands adultes : quatre fois un sujet unique, une seule fois deux exemplaires (7 et 27 mars 1953, 6 août 1953, 11 et 26 mars 1954).

On sait que $C$. longicollis est un parasite habituel de nombreuses espèces de Larus : il est cité par Georges Dubois (1938, p. 116), chez L. argentatus, L. atricilla, L. cachinnans, L. canus, L. fuscus, L. glaucus, L. marinus, L. melanocephalus et L. ridibundus.

Mes exemplaires sont conformes à la description de G. Dubois : leur longueur totale varie de 12,76 à $17 \mathrm{~mm}$. Chez un individu de $12,7 \mathrm{~mm}$., le segment antérieur mesure $1,7 \mathrm{~mm}$. et le postérieur $11 \mathrm{~mm}$. Le rapport $\frac{\text { segm. postérieur }}{\mathrm{segm} \text {. antérieur }}=6,2$. Les œufs mesurent en moyenne $120 \times 75 \mu$.

\section{Famille DiplostomatidaE Poirier 1886}

\section{DIPLOSTOMUM SPATHACEUM (Rudolphi 1819) Braun 1886}

J'ai déjà signalé en 1935 la présence de ce parasite chez les Goélands de Riou. Il s'agit là d'une espèce banale, souvent citée chez de nombreuses espèces de Larus et chez quelques autres Oiseaux ichthyophages (cf. G. Dubois 1938, p. 191).

Diplostomum spathaceum a été recueilli cinq fois à Riou : de 3 à 7 sujets par hôte adulte, localisés au début ou au milieu du dernier tiers de l'intestin.

Les caractères et les dimensions concordent en tous points avec ceux qui sont donnés par G. Dubois. Je préciserai seulement que la longueur totale de mes exemplaires est comprise entre 2,28 et $2,75 \mathrm{~mm}$. Le segment antérieur mesure 0,8 à $1,23 \mathrm{~mm}$., le postérieur 
1,4 à $1,5 \mathrm{~mm}$. Ventouse orale : 98 à $100 \mu$ (long.) sur 100 à $102 u$ (larg.), ventrale : 115 à $116 \mu$ (long.) sur 120 à $125 \mu$ (larg.). Pharynx : $90 \mu$ (long.) sur 61 à $63 \mu$. CEufs : 95 à $110 \mu \times 58,5$ à $63 \mu$.

\section{Ecologie des Trématodes de Goélands de Riou}

J.-G. Baer (1952) a bien montré que l'association du parasite et de son hôte peut être envisagée comme un problème d'écologie : chaque espèce trouve, en effet, dans les divers organes (ou portions d'organes), un biotope qui correspond à ses exigences et à ses nécessités physiologiques : ce sont ses besoins précis qui déterminent sa localisation en tel ou tel point de l'organisme de son hôte. L'étude des Trématodes de la colonie de Goélands de Riou m'a permis, en dehors des résultats purement faunistiques et biogéographiques, de dresser un relevé écologique soulignant la répartition des parasites par organe. Le tableau suivant résume ces observations :

\begin{tabular}{|c|c|c|}
\hline \multicolumn{2}{|c|}{ Vésicule biliaire $\ldots \ldots \ldots \ldots$} & $\begin{array}{l}\text { Gymnophallus deliciosns (Olsson). } \\
\text { Pachytrema calculus Looss. }\end{array}$ \\
\hline \multicolumn{2}{|c|}{ Tubes urinifères $\ldots . \ldots \ldots$} & Renicola lari Timon-David. \\
\hline \multicolumn{2}{|c|}{ Cul-de-sac conjonctival .... } & Philophthalmus sp. \\
\hline \multirow{5}{*}{ Intestin } & Tiers antérieur .. & $\begin{array}{l}\text { Rrachylecithum microtesticulatum nov. } \\
\text { sp. } \\
\text { Cardiocephalus longicollis (Rudolphi). }\end{array}$ \\
\hline & $\begin{array}{c}\text { Tiers antérieur et } \\
\text { moyen } \ldots \ldots \ldots\end{array}$ & Aporchis massiliensis nov. sp. \\
\hline & Tiers moyen .... & Mesorchis pseudoechinatus (Olsson). \\
\hline & Tiers postérieur . & $\begin{array}{l}\text { Knipowitschetrema echinatum nov. sp. } \\
\text { Stictodora sauakinensis Looss. } \\
\text { Diplostomum spathaceum (Rudolphi). } \\
\text { Brachylaemus sp. }\end{array}$ \\
\hline & Tou & yle jejuna Nicoll. \\
\hline
\end{tabular}

\section{Fréquence d'infestation}

La proportion de Goélands infestés par des Trématodes est la suivante (pourcentages calculés sur la totalité des Oiseaux examinés) : 
Echinostomatidae..........

n ............

Dicrocoeliidae ............

Galactosomatidae

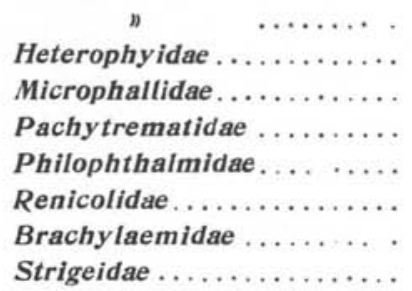

Diplostomatidae
Aporchis massiliensis sp. nov. ... Mesorchis pseudoechinatus (Olsson) Brachylecithum microtesticulatum sp. nov. ................. Knipowitschetrema echinatum sp.

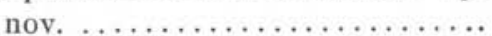
Stictodora sawakinensis Looss ... Cryptocotyle jejuna Nicoll ........ Gymnophallus deliciosus (Olsson). Pachytrema calculus Looss ...... Philophthalmus sp. ............ Renicola lari Timon-David .......

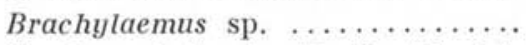
Cardiocephalus longicollis (Rudolphi) .............................. Diplostomum spathaceum (Rudolphi)
$6,8 \%$ $1,7 \%$

$1,7 \%$

$8,6 \%$ $8,6 \%$ $8,6 \%$ $3,4 \%$ $5,1 \%$ $1,7 \%$ $75 \%$ $1,7 \%$ $8,6 \%$ $8,6 \%$

\section{Influence de l'âge. Immunité}

Pour certaines espèces, les pourcentages obtenus sont bien différents quand on les calcule seulement sur les jeunes Oiseaux (d'un mois à deux mois et demi). La proportion est particulièrement élevée dans le cas de Knipowitschetrema echinatum, atteignant $24 \%$ et jusqu’à 118 exemplaires chez un seul hôte; dans le cas également de Cryptoeotyle jejuna (24\%), avec parfois plus d'un millier d'exemplaires. Ces faits témoignent de l'existence d'une certaine immunité chez les Oiseaux adultes. Des observations analogues ont déjà été faites par H. W. Stunkard (1930, p. 174), au Laboratoire de Biologie Marine de Woods-Hole, à propos de Cryptocotyle lingua (Creplin), mais l'explication exacte du phénomène est encore mal connue.

Dans le cas de Renicola lari, les statistiques donnent un résultat inverse : les adultes sont infestés dans la proportion de $80 \%$, tandis que les jeunes atteignent seulement $12 \%$. On peut supposer que cette différence est liée à une modification du régime ou, peutêtre, aux changements biochimiques relevant de l'activité endocrine chez les adultes.

\section{Abstract}

Trematoda from a herring gull rookery (Larus argentatus michaellis Naumann), at Riou Island, near Marseilles, were collected and numbered. 58 birds (41 adult from both sexes and 17 young) 
were carefully dissected. Next results were obtained : Trematoda belonging to 13 species were detected, among them 3 new are described (Aporchis massiliensis, Brachylecithum microtesticulatum, Knipowitschetrema echinatum) and several others at first time found in France.

Ecological study of these helminths show following location in various organs: Gall blader: Gymnophallus deliciosus (Olsson), Pachytrema calculus Looss; Eye, between the eyelid and the eyeball : Philophthalmus sp. ; Kidney tubules : Renicola lari T. D. ; Intestine, A) anterior part: Brachylecithum microtesticulatum n. sp. and Cardiocephalus longicollis (Rudolphi) ; B) anterior and middle part : A porchis massiliensis n. sp. ; C) middle part : Mesorchis pseudoechinatus (Olsson); D) posterior part : Knipowitschetrema echinatum n. sp., Stictodora sawakinensis Looss, Diplostomum spathaceum (Rudolphi), Brachylæmus sp.; E) whole intestine : Cryptocotyle jejuna Nicoll.

The writer gave attention to important changes concerning the composition of the helminth fauna according to the age of the birds. Some species (such as Knipowitschetrema echinatum and Cryptocotyle jejuna) occur more frequently in the young; another one, inversely (Renicolu lari), reach a percentage as high as $80 \%$ in the adult opposite to $12 \%$ in the young.

\section{BIBLIOGRAPHIE}

BAER (J. G.). - Ecology of animal parasites. Univers. of Illinois press, Urbana, 1952.

Braun (M.). - Fascioliden der Vögel. Kool. Jahrb., System., XV'I, 1902, p. 1-162, pl. I-VII.

Brinkmann (A.). - A new Trematode, Pachytrema paniceum n. sp. from the gall-blader of the Lesser Black-backed Gull (Iarus fuscus L.). Medd. Göteborgs Mnsei Zool. Andeln. Götehorgs Kungl. Vetensk.-och Vitterhets-Samhälles Handlingar. Sjätte Följden, Ser. B, 1942, 1-19.

Cillot (J.). - Matériaux pour servir à la faune des Distomes de France. Ann. Parasit. Hum. et Comp.. XXI, 1946, p. 200-201.

Dawes (Ben). - The Trematoda, Cambridge University Press, 1946.

Dietz (E.). - Die Echinostomiden der Vögel. Zool. Jahrb., Suppl. 12, 1910, p. 256-512.

Dollfus (R.-Ph.). - Sur quelques Brachylaemus de la faune française récoltés principalement à Richelieu (Indre-et-Loire). Ann. Purasit. Hum. et Comp., XII, 1934, p. 551-575, et XIII, 1935, p. 52-79.

- Sur un Distome du genre Tamerlania K. I. Skrjabin 1924 avec un catalogue des Trématodes des reins d'Oiseaux. Ann. Parasii. Hum. et Comp., XXI, 1946, p. 25-73. 
Miscellanea Helminthologica Maroccana. Quelques Trématodes, Cestodes et Acanthocéphales. Arch. Inst. Pasteur du Maroc, IV, 1951, p. 104-235.

Miscellanea Helminthologica Maroccana, XII-XXVIII. Arch. 1ust. Pasteur du Maroc, IV, 1954, p. 561-711.

Duвors (G.). - Monographie des Stigeidre (Trematoda). Mém. Soc. Neuchâtel Sc. Nat., VI, 1938, 535 p., 540 fig.

Erimov (A. S.). - Un nouveau Trématode, Philophthalmus skijabini, parasite de Larus ridibundus. Vol. Jubil. Skrjabin, Moscou, 1937, p. 149-154.

Funrmasn (O.). - Description d'un nouveau Trématode (Aporchis segmentatus n. sp.), parasite de Sterna bergii Licht. Nona Caledonia (F. Sarrasin et J. Roux), Zoologie, Wiesbaden, II, 1915, p. 213-224, fig. 1-16.

Herm de Balsac (H.). - Les Oiseaux de l'île de Riou. Rev. Franç. Ornithol., XV, $1923, \mathrm{n}^{\circ} 169$, p. 103-111.

Note complémentaire sur les Oiseaux de Riou. Ibid., XVI, 1924, n $^{\circ} 182$, p. 370-372.

et Mayaud (N.). - Nouvelles observations sur les Oiseaux de l'île de Riou. Alauda, IV, 1932, p. 85-88.

Issartschiкоу (I. M.). - Un nouveau Trématode de la famille de: Heterophyidæ (en russe). Festschrift für Knipouitsch, Moscou, 1927, p. 262-269, et fig.

Lavauden (L.) et Mourgue (M.). - Contribution à l'étude du Thalassidrome tempête dans la Méditerranée. Rev. Fr. Ornithol., X, 1918, p. 305-309.

Linton (E.). - Notes on Trematode Parasites of Birds. Proceed. Un. St. Nation. Mus., LXXIII, p. 1-36, pl. I-II.

Looss (A.). - Weitere Beiträge zur Kenntniss der Trematoden-Fauna Aegyptens zugleich Versuch einer natürlichen Gliederung des Genus Distomum Retzius. Zool. Jahrb. System, XII, 1899, p. 521-784.

Beiträge zur Systematik der Distomen. Zool. Jahrb. Syst., XXVI, 1907, p. $63-180$.

LuHe (M.). - Trematodes. In Süssimasserfauna Deutschlands, Heft. 17, 1909, 217 p.

Marcović (A.) et Garzičıć (S.). - Ueber Philophthalmus lacrymosus Braun. Zool. Anzeig., CXXVII, 1939, p. 267-270.

Monosov (P. H.). - Relations phylogénétiques entre les Trématodes de la famille des Heterophyide (en russe). Dan. U.R.S.S., LXXIV, 1950, $\mathrm{n}^{\circ} 3$.

Nicoll (W.). - A reference list of the Trematode parasites of British Birds. Parasitology, XV, 1923, p. 151-202.

Odhner (T.). - Gymnophallus, eine neue Gattung von Vogeldistomen. Zentr. Bl. f. Bakter. I. Origin., XXXI, 1900, p. 56-69.

PEREIRA (C.). - Notos helinthologicas. I. Sobre o Minuthorchis sanguineus Linton, 1928. Bol. Biol., n ${ }^{\circ} 13$, São-Paulo, 1928.

I'Unvis (G. B.). - The synonyms of the Trematode Genus Pachytrema Looss, 1907. Ann. Trop. Méd. Parasitol., XXXI, 1937, p. 457.

Rothschild (M.). - Preliminary note on the life history of Cryptocotyle jejuna Nicoll, 1907 (Trematode). Ann. Mag. Nat. Hist. London, I, Ser. II, p. 238-239.

SkRJabin (K. I.). - Trématodes des animaux et de l'Homme (en russe), T. II à IX, Moscou, 1948-1954.

Srunkard (H. W.). - The life cycle of Cryptocotyle lingua (Creplin) with notes on the physiology of the metacercaria. J. Morph. Physiol., L, 1930, 143-183. 
Timon-David (J.). - Sur une nouvelle espèce de Renicola, Trématode parasite du rein des Laridés. Bull. Inst. Océanogr. Monaco, n* 616, 1933, 16 p., 6 fig.

- Quelques observations sur les Oiseaux de l'ile de Riou et leurs parasites. Bull. Soc. Linn. Pronence, VI, 1935, 8 p., 5 fig.

Travassos (L.). - Revisao da Familia Dicrocoeliida Odhner, 1910. Monogr. Inst. Oswaldo Cruz, Rio-de-Janeiro, 1944, $\mathrm{n}^{\circ} 2,357$ p., $124 \mathrm{pl}$.

Tscherbovitch (I. A.). - Trématodes d'Oiseaux d'Extrême-Orient (en russe). Helminthol. Sb. Izd. U.R.S.S., 1946.

Wright (C. A.). - Probable relationship between the Rhodometopa group of Cercarix and the Trenatode genus Renicola Cohn. Nature, 171, 1953, p. 1072.

YAMaguti (S.). - Studies on the helminth fauna of Japan. Part 5. Trematodes of Birds III. Jap. Journ. Zool., VI, 1935, p. 159-182. Part. 25. Trematodes of Birds, IV. Ibid., 1939, VIII, p. 129-210.

- et Mrtunaga (Y.). - Trematodes of Birds from Formosa. Trans. Nat. Hist. Soc., Formosa, XXXIII, 1943, p. 312-329.

(Faculté des Sciences de Marseille

Laboratoire de Biologie animale, P.C.B.) 